\title{
Performance Analysis of Spectrum Sharing based on Bayesian Spectrum Sensing in Cognitive Radio Networks
}

\author{
Priyesh Roushan \\ (Electronics \& Communication) \\ Bharat Institute of Technology \\ Sonepat-Gohana Highway, Near \\ Mohana Police Station \\ Sonipat, Haryana 131001
}

\author{
Rupesh Kumar Jha \\ (Electronics \& Communication) \\ Bharat Institute of Technology \\ Sonepat-Gohana Highway, Near \\ Mohana Police Station \\ Sonipat, Haryana 131001
}

\author{
Piyush Chawla \\ (Electronics \& Communication) \\ Bharat Institute of Technology \\ Sonepat-Gohana Highway, Near \\ Mohana Police Station \\ Sonipat, Haryana 131001
}

\begin{abstract}
This paper addresses the comparison of spectrum sensing methods between the ARQ Retransmission technique in the context of cognitive radio with the standard detection method using Bayesian approach and drawing the inferences using the results. Two different types of spectrum sharing are taken namely conservative and aggressive and compared with legacy, Bayesian approaches. And two of them do not introduce any breakdown to the primary users who are using the spectrum and the main difference between them is that conservative does not interrupt the primary operations and whenever primary needs spectrum it will provide, but in aggressive, sometimes it may not allow the spectrum to primary user until the secondary user releases the spectrum which decreases the throughput of primary. The results have been obtained and inferences are taken.
\end{abstract}

\section{Keywords}

ARQ; Bayesian; conservative; Aggressive; spectrum sharing; spectrum sensing.

\section{INTRODUCTION}

In the context of wireless communication, it has become the fastest growing advanced numerous technology of the communication field regarding to speed, coverage, power consumption etc. In the era of wireless communication, we will have the limited spectrum which has been allotted by the FCC and the right way of using the spectrum is needed and this has motivated me to get the solution for this problem which can be mitigated by the implemented using Cognitive Radio.

With the advent of sustainable and astonishing growth in the presence of numerous wireless operating systems and the wireless services or applications operating around the world, the accessibility of quality wireless spectrum has become less limited [3]. Although, actual findings carried out at the various countries around the world gives us that most of the radio frequency spectrum is ineffectively utilized with the spectrum utilization factor mostly in the range of 5\%-50\% only [1]. Thus, the real hurdle is not the spectrum scarcity but the ineffective spectrum usage which has came from the constant spectrum allocations or reservations made by the government agencies such as TRAI in India, inflexible regulations by FCC, fixed radio functions[3], and the limited coordination between networks.
From the invention of cognitive radio by Mitola, many researchers are working on the cognitive radio related to spectrum issues in terms of spectrum sensing, spectrum sharing as well as type of channel used such as Rayleigh, Rician, AWGN etc [2].

Basic terminology regarding the cognitive radio functionalities are given below:

Spectrum is defined as the band of frequencies that are allocated for the particular users in a particular region [5][7].

Primary users are the users who can use the spectrum whenever they want and they have no restrictions and are called as licensed users in terms of wireless communication and these are the rightful users of the spectrum or network. Similarly, secondary users are the users who can use only when spectrum is free and returns the spectrum channels when primary user needs and also called as unlicensed users.

Cognitive Radio is an intelligent software defined radio that monitors its surroundings continuously and varies its parameters accordingly to allot spectrum channels to the secondary users when needed. Underlay Cognitive radio is called only if the unlicensed user transmits in the allotted spectrum of a primary user without degrading the performance of the primary user.

Ad-hoc network is the wireless network where mobile nodes are connected with each other without any centralized infrastructure as in wireless mobile communication [10].

In [12], they have studied extensively on spectrum sensing and sharing methods and developed an ARQ model and proposed spectrum sharing types such as conservative, aggressive and compared the throughputs of the duo by varying secondary power as well as SIR(signal to interference ratio), nominal spectral efficiency. In [13], they worked on the energy detection technique i.e, Bayesian detection in which sample energy calculated iteratively and also they compared all the energy detection techniques.

In this paper, as an extension to [12], we have compared the results of [12] with Bayesian energy detector and inferences have been drawn. Coming to our topic, first of all we have implemented the base paper [12] and thanks to the authors for doing a great job and I have tried to find some extension or modification to that paper, since cognitive radio is an vast topic and I have tried to compare the results of ARQ 
retransmission technique in cognitive radio [12] with the standard and most efficient technique for finding energy in the signal which is Bayesian energy detection method [13].

The body of the paper has been organized as follows section II introduces the model of the system which is depicted from [12]. In section III, three spectrum sharing schemes are mentioned which are aggressive, conservative and legacy which uses ACK/NACK feedback signals are taken [12]. Then Bayesian energy detection has been introduced. Afterwards the throughput analysis of above mentioned schemes as well as Bayesian, conventional detection methods are given in section IV. Finally some simulation results have been given and necessary inferences are drawn using those results which are given in section $\mathrm{V}$ and VI respectively.

\section{SYSTEM MODEL}

The system model is entirely based on the ad-hoc networks and this model is mainly applicable to the mobile devices which are extremely taken in a stationary environment e.g., ad-hoc environment scenario in an office. In our paper, we will assume that the channel is experiencing slow fading which is extending through many transmission time intervals. We know about the CSI- channel state information, where it tells us about the characteristics of the channel, whether it is busy or unoccupied and depending on that information, we can allot the channels to the primary or secondary users. The channel gains of the channel are shown by ' $g_{i j}$ ' from transmitter $i$ to receiver $j$, where the subscript value $i$ denotes the primary and $\mathrm{j}$ denotes the secondary. Channel gains will obey the exponential distribution with the mean $\lambda$. The system model that has proposed has been given in figure.1.

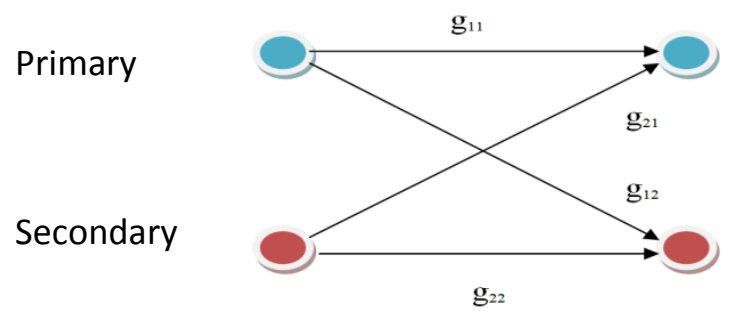

Fig 1: system model[12]

In this paper, we have simply taken a simple ad-hoc network where ARQ based spectrum sharing taking place without using the traditional CSI (channel state information) at the secondary transmitter. ARQ means automatic repeat request and the mechanism used in the process is whenever the source node transmits a message to the destination user, if the message received successfully by the destination then it sends ACK-acknowledgement signals back to the sender such that sender assumes that message reached successfully [8][9][11].

If at all message doesn't reached the destination, then it will send NACK-negative acknowledgement signal to the sender such that sender assumes that message should be retransmitted.

We are now considering another two additional things in this process cycle [12]:

(1) Each of the primaries ARQ mechanism will now carries the information regarding the channel gains that has been taking place in multiple time intervals.
(2) Each of the secondary transmission creates interference on the primary user in the network; therefore the following ARQ now carries further information back to the secondary regarding the relative strength of the primary and values of the cross channel coefficients.

In the aggressive sharing, the secondary will only transmit whenever it has possible to do so without sending the primary into breakdown, even though it will degrade the performance of the primary throughput [6].

In the Conservative sharing, the secondary will only transmit when there has been no negative effect on the primary throughput. We can now determine the probing or searching and as well discovery mechanism. The probing of the system have determined by the secondary transmission decisions only [12].

For our clarity, we will use the following notations that will used to combine the transmission modes of the primary user and as well as the secondary cognitive user.

$\mathrm{T} 0=\{$ primary transmits brand new packet; where secondary keeps idle $\}$

$\mathrm{T} 1=\{$ primary repeats the older packet; secondary keeps idle $\}$

$\mathrm{T} 2=\{$ primary transmits the another new packet; secondary also transmits $\}$

$\mathrm{T} 3=\{$ primary repeats the older packet; secondary transmits $\}$.

Using the above notations, the discovery process for the secondary user is relatively simple and easy to grab quickly to understand, and has been shown in the flowcharts of Figures 2 and 3. The algorithm will be initialized from the root or base of the tree, and proceeds to a leaf or edge. Throughout this process, the secondary will make transmission decisions and observes and analyzes the ACK/NACK from the primary, until it will determine which of the six regions it is to be operating in. The searching and the channel detection for each of the six operating regions are outlined and mentioned below.

Region (S1) is discovered by getting one ACK, searching the primary channel (T2) and receiving another ACK. This will indicate that primary channel only supports the rate in only one transmission despite with any interference.

Region (S2) is discovered by getting the first ACK, then the secondary probing in two successive intervals (T2, T3) and also getting an NACK followed by an ACK. This indicates that the primary channel will support its rate in only one interference-free transmission, but in the presence of interference it may need two transmissions to succeed.

Region (S3) is discovered by getting an ACK, then probing will done in two such successive intervals (T2, T3) and receiving the two NACKs. This will indicate that the primary channel can supports the rate in only one interference-free transmission, but in the presence of interference it will be in the outage even with the retransmission of the packet.

Region (S4) is discovered when the following sequence happens: getting an initial NACK (which, can recall that, was under no interference), and the secondary will be staying silent or idle and will get an ACK (now we know the primary will get through in two transmissions if left alone). On the next transmission the secondary also stays silent or idle but listen's an NACK (as expected), the next time the secondary transmits (T3) and hears an ACK. This tells us that the primary channel can only support the rate in two (but not one) interference-free transmissions; it can also be succeeded in two reliable 
transmissions as long as only one of the transmissions will be subjected to the interference.

Region (S5) is discovered by going through the same sequence as the case described above, but however, in the last stage Instead of an ACK a NACK is received, showing that despite with all care the secondary cannot transmit. This indicates that the primary channel supports the rate in two (but not one) interference-free reliable transmissions, and that it cannot support its rate with interference (even on one of its two transmissions).

Region (S6) is discovered by the secondary staying idle for the two reliable transmission intervals. When two successive back to back NACKs are received by the primary, it is known that the primary is in breakdown even with the absence of secondary [12].

The detection of the operating region for the aggressive can be described as a systematic way as shown in the Figure 2. Starting from the root or base of the tree, the secondary stays idle for the first transmission and observes the primary ACK/NACK. Each of the six detection cases mentioned above fetches a route from the root of the tree to one of the six leaves or edges of the tree. The flow charts for the aggressive as well as the conservative spectrum sharing have been given in the following figures 2 and 3 .

The outcomes S2, S3 from the aggressive sharing which has been resulted in the primary throughput degradation are now changed into a single outcome S2' in conservative sharing as shown the in figure 3 , where no secondary transmission is allowed to access the channel.

It has to be noted that either the spectrum sharing techniques such as aggressive or conservative does not produces any primary breakdown. The main difference between them is that the aggressive may occasionally will used to slow down the primary throughput by forcing it to use two time slots instead of one transmission interval. This is a slightly different form of degradation, where the primary is slightly degraded in throughput because the secondary may enforces on the primary (a small amount of) additional breakdown.

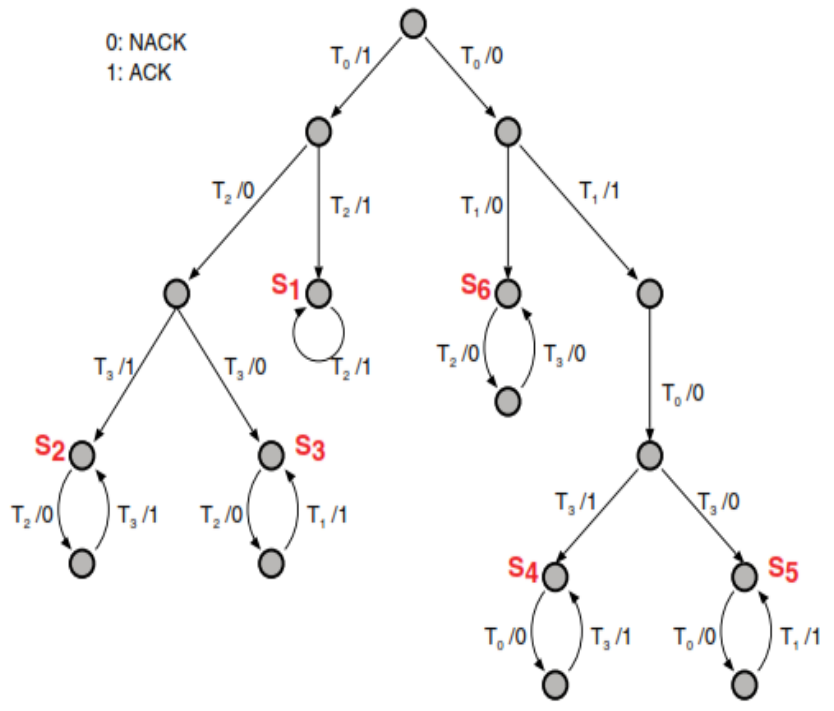

Fig 2: Flow chart for the aggressive spectrum sharing [12]

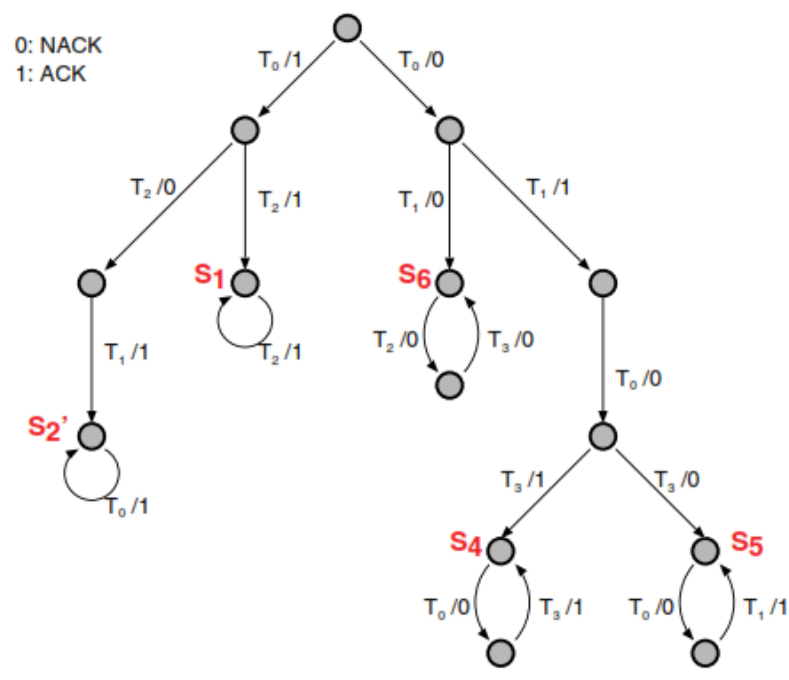

Fig 3: Flow chart for the conservative spectrum sharing [12]

The probability of the channel gains residing in each of the six operating regions are formulated and are given below.

$\mathrm{P}\left(\mathrm{S}_{\mathrm{n}}\right)$ - Probability of the channel gains in $\mathrm{n}^{\text {th }}$ region.

$$
P\{S 1\}=P\left\{\frac{g_{11} P p}{1+g_{21} P s} \geq \gamma_{p}\right\}=\frac{\lambda_{11} P p e^{-\frac{\gamma_{p}}{\lambda 11 P p}}}{\lambda_{21} \text { Ps } \gamma_{p}+\lambda_{11} P p}
$$

Where, $g_{11}, g_{21}, g_{22}, g_{12}$ are the respective channel gains.

Ps- secondary transmitted power.

Pp- primary transmitted power.

We use the following related considerations for our understandings and the flexibility,

$\gamma_{\mathrm{p}} \triangleq 2^{\mathrm{Rp}}-1$ and $\gamma_{\mathrm{s}} \triangleq 2^{\mathrm{Rs}}-1$

And Rp- the related Nominal spectral efficiency of the respective primary consumer in bits/sec/Hz,

Rs- The related Nominal spectral efficiency of the respective secondary consumer in bits/sec/Hz.

$\lambda_{11}, \lambda_{21}$ - are the respective mean of the channel propagation gains.

Probability of the channel gain in region S2 is given by

$$
\begin{gathered}
P\{S 2\}=P\left\{\frac{g_{11} P p}{1+g_{21} P s}<\gamma_{p}, g_{11} P p \geq \gamma_{p} \frac{g_{11} P p}{1+g_{21} P s}\right. \\
\left.\geq \gamma_{p} / 2\right\} \\
=\frac{\lambda_{21} P s \gamma_{p} e^{-\frac{\gamma_{p}}{\lambda 11 P p}}}{\lambda_{21} P s \gamma_{p}+\lambda_{11} P p}-\frac{\lambda_{21} P s \gamma_{p} e^{-\left(\frac{\gamma_{p}}{\lambda_{11} P p}+\frac{1}{\lambda_{21 P s}}\right)}}{\lambda_{21} P s \gamma_{p}+2 \lambda_{11} P p}
\end{gathered}
$$

Probability of the channel gain in region $\mathrm{S} 3$ is given by

$$
\begin{aligned}
& \mathrm{P}\{\mathrm{S} 3\}=\mathrm{P}\left\{\mathrm{g}_{11} \mathrm{Pp} \geq \gamma_{\mathrm{p}}, \frac{\mathrm{g}_{11} \mathrm{Pp}}{1+\mathrm{g}_{21} \mathrm{Ps}}<\frac{\gamma_{\mathrm{p}}}{2}\right\} \\
& =\frac{\lambda_{21} \mathrm{Ps} \gamma_{\mathrm{p}} \mathrm{e}^{-\left(\frac{\gamma_{\mathrm{p}}}{\lambda_{11} \mathrm{Pp}}+\frac{1}{\lambda_{21} \mathrm{Ps}}\right)}}{\lambda_{21} \mathrm{Ps} \gamma_{\mathrm{p}}+2 \lambda_{11} \mathrm{Pp}}
\end{aligned}
$$

Probability of the channel gain in region S4 is given by

$$
\mathrm{P}\{\mathrm{S} 4\}=\mathrm{P}\left\{\mathrm{g}_{11} \mathrm{Pp}+\frac{\mathrm{g}_{11} \mathrm{Pp}}{1+\mathrm{g}_{21} \mathrm{PS}} \geq \gamma_{\mathrm{p}}, \mathrm{g}_{11} \mathrm{Pp}<\gamma_{\mathrm{p}}\right\}
$$




$$
=\mathrm{e}^{-\frac{\gamma_{\mathrm{p}}}{\lambda_{11 \mathrm{Pp}}}\left(\varphi\left(\wedge_{21} \mathrm{Ps}, \frac{\lambda_{11} \mathrm{Pp}}{\gamma_{\mathrm{p}}}\right)-1\right)}
$$

Probability of the channel gain in region S5 is given by

$$
\begin{gathered}
\mathrm{P}\{\mathrm{S} 5\}=\mathrm{P}\left\{\mathrm{g}_{11} \mathrm{Pp} \geq \frac{\gamma_{\mathrm{p}}}{2}, \mathrm{~g}_{11} \mathrm{Pp}+\frac{\mathrm{g}_{11} \mathrm{Pp}}{1+\mathrm{g}_{21} \mathrm{Ps}}<\gamma_{\mathrm{p}}\right\} \\
=\mathrm{e}^{-\frac{\gamma_{\mathrm{p}}}{2 \lambda_{11} \mathrm{Pp}}}-\mathrm{e}^{-\frac{\gamma_{\mathrm{p}}}{\lambda_{11 \mathrm{Pp}}}\left(\varphi\left(\lambda_{21} \mathrm{Ps}, \frac{\lambda_{11} \mathrm{Pp}}{\gamma_{\mathrm{p}}}\right)\right)}
\end{gathered}
$$

Probability of the channel gain in region S6 is given by

$$
\mathrm{P}\{\mathrm{S} 6\}=\mathrm{P}\left\{\mathrm{g}_{11} \mathrm{Pp}<\gamma_{\mathrm{p}} / 2\right\}=1-\mathrm{e}^{-\frac{\gamma_{\mathrm{p}}}{2 \Lambda_{11} \mathrm{Pp}}}
$$

Where, $\varphi(y, z) \triangleq \int_{0}^{+\infty} \mathrm{e}^{\frac{1}{(2+y t) z}-t} d t$.

The inference between aggressive and conservative SHARP is that whether the secondary consumer is allowed to delay the primary's transmission cycle or not. In the conservative scheme, Region S2 and S3 are combined to ' $\mathrm{S}_{2}^{1}$ ' with and the secondary consumer has not been allowed to transmit in this concerned related region. [12]

$$
\begin{gathered}
P\left\{s_{2}^{1}\right\}=P\left\{\frac{g_{11} P p}{1+g_{21} P s}<\gamma_{p}, g_{11} P p \geq \gamma_{p}\right\} \\
=\frac{\lambda_{21} P s \gamma_{p} e^{-\left(\frac{\gamma_{p}}{\lambda_{11} P p}\right)}}{\lambda_{21} P s \gamma_{p}+\lambda_{11} P p}
\end{gathered}
$$

\section{THROUGHPUT AND OUTAGE PROBA- BILITY ANALYSIS}

The primary packet is sent by only one transmission cycle in the Region S1 and two cycles in the other mentioned SNR regions. Except in Region S6, the packet is the only successfully decoded at the primary receiver. As a result, the throughput for the primary consumer in the aggressive SHARP is given as

$$
\mathrm{G}_{\mathrm{p}}^{\mathrm{A}}=\operatorname{RpP}\{\mathrm{S} 1\}+\frac{\mathrm{Rp}}{2} \sum_{\mathrm{i}=2}^{5} \mathrm{P}\{\mathrm{Si}\}
$$

Where, the ' $A$ ' denotes the aggressive and $R_{p} / 2$ is taken due to the fact that it consumes two consecutive transmission cycles.

Accordingly, the throughput of the secondary consumer in aggressive sharing can also be derived and given as,

$$
\mathrm{G}_{\mathrm{s}}^{\mathrm{A}}=\left(\begin{array}{c}
\mathrm{R}_{\mathrm{s}}(\mathrm{P}\{\mathrm{S} 1\}+\mathrm{P}\{\mathrm{S} 2\}+\mathrm{P}\{\mathrm{S} 6\}) \\
+\frac{\mathrm{Rs}}{2}(\mathrm{P}\{\mathrm{S} 3\}+\mathrm{P}\{\mathrm{S} 4\}) \\
-\mathrm{P}^{\mathrm{OS}\}}
\end{array}\right)\{1
$$

Apart from exploiting and digging the transmission opportunities for the secondary in the regions S1 and S4 which makes no harmful effect to the existing primary operating system, the secondary consumer may slows down the primary by forcing it to use two transmission cycles instead of one in the Regions S2 and S3. In addition to these, the secondary is allowed to transmit when there is two interference-free transmissions are not good enough to support the primary consumer in the Region S6.
The conservative sharing aims to avoid any negative adverse effect on the primary consumer by allowing the secondary to transmit only when the channel is good enough to support simultaneous communication for both the primary as well as the secondary [12].

The conservative scheme also precludes the transmission in the region $\mathrm{S}_{2}^{1}$ (i.e., $\mathrm{S} 2 \cup \mathrm{S} 3$ ), and leaves the primary operation alone. Consequently, the throughput of the primary as well as the secondary in the conservative sharing are given by

$$
\begin{array}{r}
\mathrm{G}_{\mathrm{p}}^{\mathrm{C}}=\mathrm{Rp}\left(\mathrm{P}\{\mathrm{S} 1\}+\mathrm{P}\left\{\mathrm{S}_{2}^{1}\right\}\right)+\frac{\mathrm{Rp}}{2}(\mathrm{P}\{\mathrm{S} 4\}+\mathrm{P}\{\mathrm{S} 5\}) \\
\mathrm{G}_{\mathrm{s}}^{\mathrm{C}}=\left(\mathrm{R}_{\mathrm{s}}\left(\mathrm{P}\{\mathrm{S} 1\}+\frac{1}{2} \mathrm{P}\{\mathrm{S} 4\}+\mathrm{P}\{\mathrm{S} 6\}\right)\right)\{1 \\
-\mathrm{P}^{\mathrm{OS}\}}(11)
\end{array}
$$

Bayesian energy detection can be used for the mathematical basic function of cognitive radio, and according to the Bayesian the power that has been detected related to SNR and noise power is given by [12]

$p_{d}$

$=Q\left\{\log \left(e_{\text {thresh }}\right)-2 N\left(S N R^{2)} \mid S N R \sqrt{N(2+8 S N R)}\right\}\right.$

Where, $e_{\text {thresh }}={ }^{p_{r 0}} / p_{r 1}$ and $p_{r 0,=0.85,} p_{r 1=0.15}$ are the assumed received powers.

SNR- signal to noise ratio, $\mathrm{N}$-noise power and assumed to be a constant value ' 10 '.

Where, ' $\mathrm{Q}(\mathrm{x})$ ' function is given as

$$
Q(x)=\frac{1}{\sqrt{2 \pi}} \int_{x}^{\propto} \exp \left(-t^{2} / 2\right) d t
$$

The throughput for the Bayesian detection sharing related to the Bayesian detected power is given by [12]

$$
g_{b}=\log \left(1+p_{d} S N R\right) /_{S N R}
$$

On varying or changing the $P^{O S}$ in the below equations given, we can calculate for the different interference cancellation schemes such as PIC (perfect interference cancellation), SBIC (single block interference cancellation), and BIC (block interference cancellation) regions for both aggressive and conservative sharing.

The secondary consumer will only allow transmitting only when the interference from the primary is perfectly or absolutely cancelled at the secondary receiver [12]. The corresponding obtained outage probability for the secondary has been given as

$$
\mathrm{P}^{\mathrm{OS}}=\mathrm{P}_{\mathrm{PIC}}^{\mathrm{OS}}=1-\mathrm{e}^{-\frac{\gamma_{\mathrm{S}}}{\lambda_{22} \mathrm{PS}}}
$$

Where, the superscript indicates the 'Outage for Secondary' and the subscript 'PIC' denote 'Perfect Interference Cancellation.

The result obtained is based on the condition that, the secondary receiver doesn't locate neither far away nor close to the primary transmitter.

We have taken the interference into the consideration, and allowed the secondary to coexist with the interference from the primary user. Specifically, the secondary tries to equalize 
the interference from primary in the first place. If the received signal-to-interference-plus-noise ratio (SINR) is greater than $\gamma \mathrm{p}$, the interference is considered to be equalized from the received signal. Otherwise, the secondary will simply treats the interference as the additional background noise. In this method the secondary receiver attempts to cancel the interference signals using only the information received in only one block, hence this approach has been denoted as single-block interference cancellation [12].

Then the outage probability of the secondary is then given as for single-block interference cancellation.

$$
\begin{gathered}
\mathrm{P}^{\mathrm{OS}}=\mathrm{P}_{\mathrm{SBIC}}^{\mathrm{OS}}=\mathrm{P}\left\{\frac{\mathrm{g}_{11} \mathrm{Pp}}{1+\mathrm{g}_{22} \mathrm{Ps}} \geq \gamma_{\mathrm{p}}, \mathrm{g}_{22} \mathrm{Ps}<\gamma_{\mathrm{s}}\right\} \\
+\mathrm{P}\left\{\frac{\mathrm{g}_{12} \mathrm{Pp}}{1+\mathrm{g}_{22} \mathrm{Ps}}<\gamma_{\mathrm{p}}, \frac{\mathrm{g}_{22} \mathrm{Ps}}{1+\mathrm{g}_{12} \mathrm{Pp}}<\gamma_{\mathrm{s}}\right\}
\end{gathered}
$$

It has been noticed in that the secondary receiver is able to buffer the initially received packet and attempts to equalize the interference came from the primary as a whole when both duplicate copies in the two transmission slots are received, i.e. can be called as Backward Interference Cancellation (BIC).

In this type of manner, the throughput of the secondary consumer can also be improved further. The difference lies in the fact that BIC requires the secondary receiver to eavesdrop (silently listening) on the ARQ feedbacks that has came from the primary receiver so that the decoder can also recognize whether the interference from the primary is the repeating duplicate copy or a totally new packet. If this ARQ information is made available at the secondary receiver, then the outage probability for the secondary consumer can also be further can be improved as follows:

$$
\mathrm{P}^{\mathrm{OS}}=\left\{\begin{array}{lr}
\mathrm{P}_{\mathrm{SBIC}}^{\mathrm{OS}} & \mathrm{S} 1 \\
\mathrm{P}_{\mathrm{BIC} 1}^{\mathrm{OS}} & \mathrm{S} 2 \text { AND S6 } \\
\mathrm{P}_{\mathrm{BIC} 2}^{\mathrm{OS}} & \mathrm{S} 3 \text { AND S4 } \\
\mathrm{N} & \mathrm{S} 5 \text { AND } \mathrm{S}_{2}^{1} \\
\hline \mathrm{A} & \mathrm{s}
\end{array}\right.
$$

Where, the detailed wanted explanations for the above given equation are given below:

- When in the operating region $S 1$, the primary packet has been sent only once from the primary transmitter.

- When in operating region $S 2$ and $S 6$, the primary packet will be going to be repeated, and the secondary can able to buffer in the primary message and tries to decode it only after receiving the two duplicate copies of the same transmitted message. In the meanwhile, the secondary transmitter sends packets (information) in both slots. The outage probability of this BIC scheme has been given below:

$$
\begin{gathered}
\mathrm{P}_{\mathrm{BIC} 2}^{\mathrm{OS}}=\mathrm{P}\left\{\mathrm{g}_{12} \mathrm{Pp}+\frac{\mathrm{g}_{12} \mathrm{Pp}}{1+\mathrm{g}_{22} \mathrm{Ps}} \geq \gamma_{\mathrm{p}}, \mathrm{g}_{22} \mathrm{Ps}<\gamma_{\mathrm{s}}\right\} \\
+\mathrm{P}\left\{\mathrm{g}_{12} \mathrm{Pp}+\frac{\mathrm{g}_{12} \mathrm{Pp}}{1+\mathrm{g}_{22} \mathrm{Ps}}<\gamma_{\mathrm{p}}, \frac{\mathrm{g}_{22} \mathrm{Ps}}{1+\mathrm{g}_{12} \mathrm{Pp}}\right. \\
\left.<\gamma_{\mathrm{s}}\right\}
\end{gathered}
$$

- $\quad$ When in the operating regions $S 3$ and $S 4$, the primary packet will sent twice, but the secondary consumer utilizes the transmission slot only once (either the first as in $S 3$ or the second as in S4). Therefore, the corresponding outage probability with the BIC can be obtained as given below:

$$
\begin{gathered}
\mathrm{P}_{\mathrm{BIC} 2}^{\mathrm{OS}}=\mathrm{P}\left\{\mathrm{g}_{12} \mathrm{Pp}+\frac{\mathrm{g}_{12} \mathrm{Pp}}{1+\mathrm{g}_{22} \mathrm{Ps}} \geq \gamma_{\mathrm{p}}, \mathrm{g}_{22} \mathrm{Ps}<\gamma_{\mathrm{s}}\right\} \\
+\mathrm{P}\left\{\mathrm{g}_{12} \mathrm{Pp}+\frac{\mathrm{g}_{12} \mathrm{Pp}}{1+\mathrm{g}_{22} \mathrm{Ps}}<\gamma_{\mathrm{p}}, \frac{\mathrm{g}_{22} \mathrm{Ps}}{1+\mathrm{g}_{12} \mathrm{Pp}}<\gamma_{\mathrm{s}}\right\}
\end{gathered}
$$

- When in the operating regions $S 5$ and $S_{2}^{1}$ (for conservative SHARP), the secondary transmitter will used to be remains silent or idle.

\section{EXPERIMENTAL RESULTS}

In this section, I have described the results of the various schemes that have been described above has been validated and compared the spectrum sharing schemes such as aggressive, conservative, legacy with the standard energy technique Bayesian detector and the inferences has been drawn by taking the simulation results.

The probability of the six regions mentioned in the above system model has been compared graphically against the secondary transmit power (in decibels). As shown in the below figure, maximum probability of the regions is equal to unity (nearly equal to 1 ).

The probability of Region $S 6$ is a constant due to the fact that it is independent of secondary transmit power Ps. Moreover, it can be seen that the probabilities of Region $S 1$ and $S 4$ both decrease as $P s$ increases.

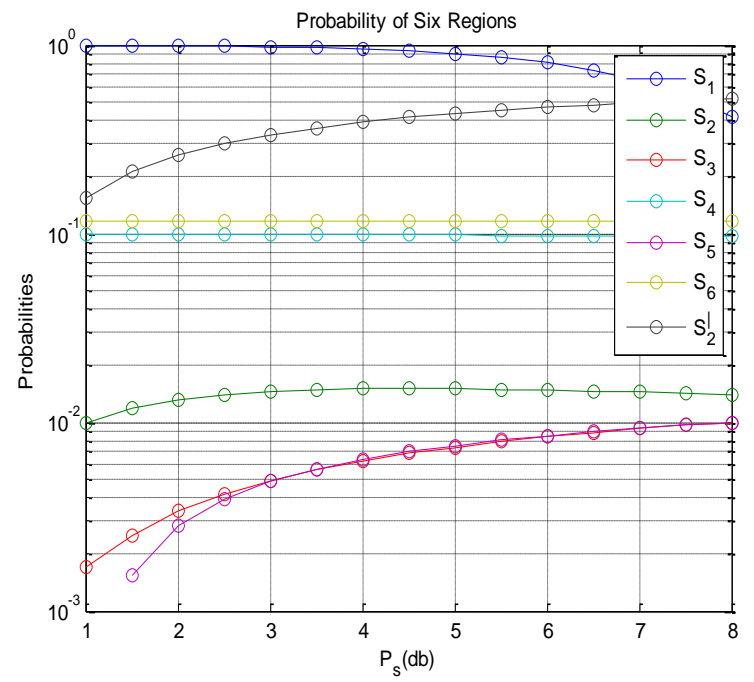

Fig 4: probabilities of six regions against secondary transmit power

The mentioned below figure compares the throughput of the different mentioned spectrum sharing techniques such as aggressive, conservative and legacy schemes and has been compared graphically in Matlab by varying the SNR (signal to noise ratio) values which has been depicted from [12] and has been compared with the mentioned with the described spectrum sharing techniques such as Bayesian energy detection techniques [13]. 


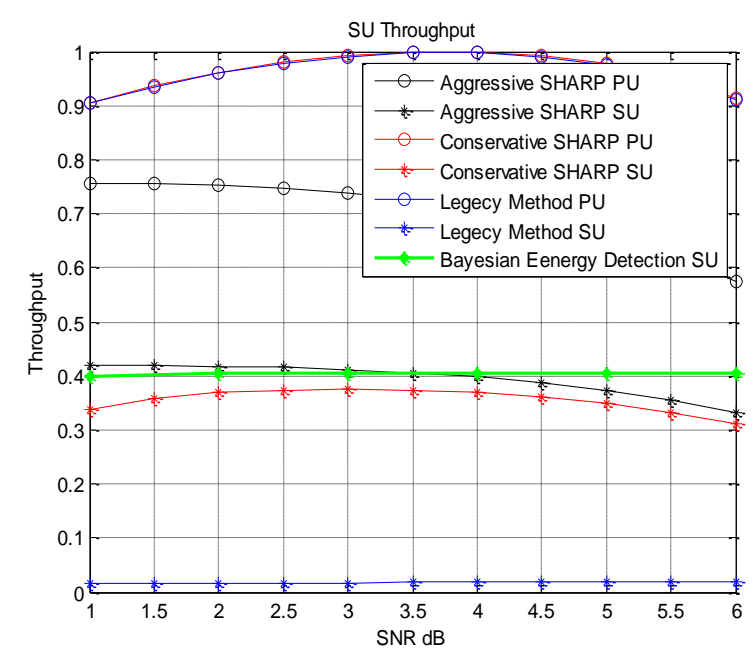

Fig 5: throughput of secondary against SNR corresponding to all spectrum sharing techniques

The secondary user throughput results with the three interference cancellation schemes mentioned are given below. We vary the rate outage threshold for the primary and observe that all three schemes are similar to each other in terms of the secondary user throughput when the rate threshold $R p$ (nominal spectral efficiency of the primary) is low, but the single-block interference cancellation scheme has some difficulties when the requirement for the decoding gets higher.

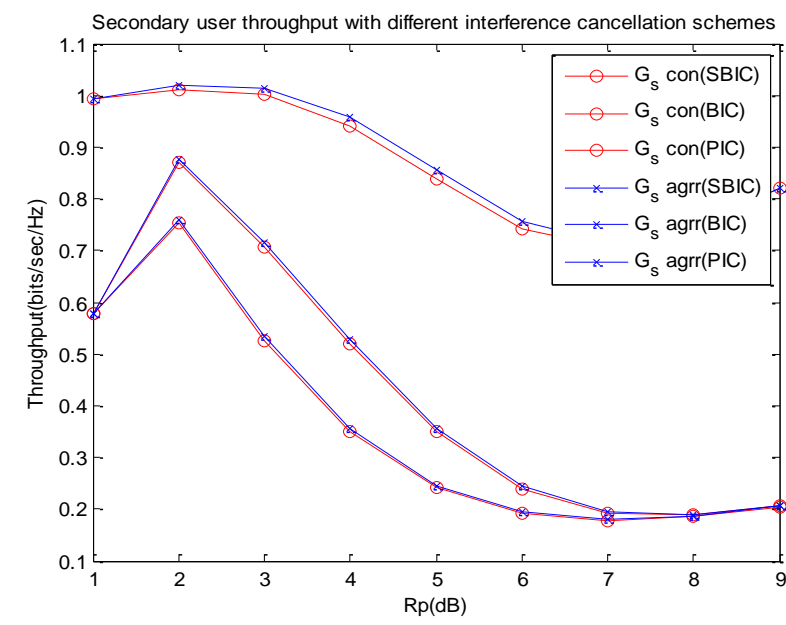

Fig 6: secondary user throughput against $R p$ in different cancellation schemes.

\section{CONCLUSION}

The key contribution of this paper was about the research process is centred on the spectrum sharing techniques such as aggressive, conservative and legacy which are considered from [12] and throughputs of the considered techniques has been compared with the proposed Bayesian energy detection . Before the throughput calculation, first of all we have divided the all possible regions into six regions as mentioned in the system model and the respected probabilities of those regions has been calculated. Then depending on these probabilities, we have calculated the throughputs of the secondary as well as primary with respect to the mentioned spectrum sharing techniques has been calculated, also throughputs of the different interference cancellation schemes mentioned in section III has also been calculated. Finally results are obtained for the throughputs comparison of the different mentioned techniques.

\section{ACKNOWLEDGMENTS}

This paper has been written with the kind assistance, guidance and active support of our department who have helped us in this work. We would like to thank all the individuals whose encouragement and support has helped us for the completion of this work.

\section{REFERENCES}

[1] "Spectrum policy task force," Federal Communications Commission (FCC), ET Docket No. 02-135, Tech. Rep., Nov. 2002.

[2] M. Gastpar, "On capacity under receive and spatial spectrum-sharing constraints," IEEE Trans. Inf. Theory, vol. 53, no. 2, pp. 471-487, Feb. 2007.

[3] A. Ghasemi and E. S. Sousa, "Fundamental limits of spectrum-sharing in fading environments," IEEE Trans. Wireless Commun., vol. 6, no. 2, pp. 649-658, Feb. 2007.

[4] K. B. Letaief and W. Zhang, "Cooperative communications for cognitive radio networks," Proc. IEEE, vol. 97, no. 5, pp. 878-893, May2009.

[5] Q. Zhang, J. Jia, and J. Zhang, "Cooperative relay to improve diversity in cognitive radio networks," IEEE Commun. Mag., vol. 47, no. 2, pp. 111-117, Feb. 2009.

[6] S. Srinivasa and S. A. Jafar, "The throughput potential of cognitive radio: a theoretical perspective," IEEE Commun. Mag., vol. 45, no. 5, pp. 73-79, May 2007.

[7] A. Goldsmith, S. A. Jafar, I. Maric, and S. Srinivasa, "Breaking spectrum gridlock with cognitive radios: an information theoretic perspective," Proc. IEEE, vol. 97, no. 5, pp. 894-914, May 2009.

[8] K. Eswaran, M. Gastpar, and K. Ramchandran, "Bits through ARQs:spectrum sharing with a primary packet system," in Proc. 2007 IEEE Int. Symp. on Information Theory, pp. 2171-2175.

[9] M. Levorato, U. Mitra, and M. Zorzi, "Cognitive interference management in retransmission-based wireless networks," in Proc. 2009 Allerton Conf. on Commun. Control and Computing, pp. 94-101.

[10] R. Zhang, "On active learning and supervised transmission of spectrum sharing based cognitive radios by exploiting hidden primary radio feedback," in Proc. 2009 IEEE Global Commun. Conf., pp. 1-5.

[11] R. A. Tannious and A. Nosratinia, "Cognitive radio protocols based on exploiting hybrid ARQ retransmissions," IEEE Trans. Wireless Com-mun., vol. 9, no. 9, pp. 2833-2841, Sep. 2010.

[12] James C. F. Li, Wei Zhang, Aria Nosratinia," SHARP: Spectrum Harvesting with ARQ Retransmission and Probing in Cognitive Radio" IEEE transactions on communications, vol. 61, no. 3, march 2013.

[13] Ahmet Gokceoglu , Robert Piché, and Mikko Valkama1 "Bayesian Approach to Spectrum Sensing for Cognitive Radio Applications" CROWNCOM 2012, June 18-20, Stockholm, Sweden. 